\title{
Sirtuin 1 Promotes Hyperoxia-Induced Lung Epithelial Cell Death Independent of NF-E2-Related Factor 2 Activation
}

\author{
Haranatha R. Potteti*, Subbiah Rajasekaran*, Senthilkumar B. Rajamohan*, Chandramohan R. Tamatam, \\ Narsa M. Reddy, and Sekhar P. Reddy \\ Division of Developmental Biology and Basic Research, Department of Pediatrics, University of Illinois at Chicago, Chicago, Illinois
}

\begin{abstract}
Lung epithelial cell damage accompanied by death is a cardinal feature of toxicant- and prooxidant-induced acute lung injury. The transcription factor nuclear factor (erythroid-derived 2)-like 2 (NEF2L2 or NRF2) activates several antioxidant enzymes (AOEs) and prosurvival genes in response to oxidant stress, and its deficiency enhances susceptibility to hyperoxic lung injury and other oxidantinduced lung pathologies. Sirtuin 1 (SIRT1) regulates cell growth and survival in response to both physiological and pathological stresses by selectively deacetylating multiple proteins required for chromatin remodeling and transcription; therefore, we sought to examine potential SIRT1-NRF2 cross-talk in the regulation of AOE expression during hyperoxia-induced lung epithelial cell death. Unexpectedly, pharmacological inhibition or small interfering RNA-mediated depletion of SIRT1 caused a reduction in cell death, accompanied by reduced levels of NRF2-dependent AOE expression in chronic hyperoxia. NRF2 acetylation was markedly and transiently higher in cells exposed to acute $(6 \mathrm{~h})$ hyperoxia. Sirtinol blocked this acute effect, but NRF2 acetylation was low or undetectable in cells exposed to chronic hyperoxia (24-36 h) both with and without sirtinol. SIRT1 activation by resveratrol augmented hyperoxia-induced death in cells
\end{abstract}

with NRF2 deficiency. SIRT1 inhibition or depletion led to a reduced activation of the cell-death executioner caspase 3, whereas caspase inhibition prevented death. Consistent with these results, sirtinol attenuated hyperoxia-induced lung alveolar permeability and toxicity in vivo. Collectively, these results reveal that, in chronic hyperoxia, SIRT1 promotes hyperoxia-induced lung epithelial cell damage and death by altering pro- and antiapoptotic balance, not by dampening optimal NRF2-dependent AOE expression.

Keywords: acute lung injury; oxidative stress; antioxidants; deacetylases; airway epithelium

\section{Clinical Relevance}

Persistent damage and death of lung epithelial cells is associated with abnormal lung tissue repair and inflammation after hyperoxic lung injury. Our studies indicate sirtuin 1 as a critical positive regulator of hyperoxic epithelial death; thus, targeting this deacetylase may be useful to mitigate cell death induced by chronic hyperoxia.
Oxidative stress has been implicated in both acute lung injury and its severe form, acute respiratory distress syndrome, both of which are clinical disorders with significant morbidity and mortality. Hyperoxia (oxygen supplementation) is used to support patients who are critically ill, but this therapy can contribute to or perpetuate preexisting lung injury in patients who are critically ill and in laboratory animals (1). Alveolar epithelial and endothelial cell death and/or their abnormal repair after injury can lead to impairment of respiratory function and pathogenesis (2). Nuclear factor (erythroidderived 2)-like 2 (NEF2L2 or NRF2) is a transcription factor that confers cytoprotection against prooxidant insults. Nrf2 deficiency increases susceptibility to hyperoxic lung injury (3) and impairs the resolution of lung inflammation after

(Received in original form February 18, 2014; accepted in final form October 1, 2015)

*These authors contributed equally to this article.

This work was supported in part by National Institutes of Health grants HL66109 and ES11863 (S.P.R.).

Author Contributions: H.R.P., S.R., S.B.R., and S.P.R. were involved in the conception, delineation of hypotheses, and design of the study, as well as the analysis and interpretation of data. H.R.P., S.R., and S.B.R. performed experiments. C.R.T. and N.M.R. helped with the mouse studies and data acquisition. H.R.P., S.R., S.B.R., and S.P.R. wrote the manuscript. All the authors read and approved the final manuscript.

Correspondence and requests for reprints should be addressed to: Sekhar P. Reddy, Ph.D., Department of Pediatrics, University of Illinois at Chicago, M/C 856, 830 South Wood Street, Chicago, IL 60612. E-mail: sreddy03@uic.edu

This article has an online supplement, which is available from the issue's table of contents at www.atsjournals.org

Am J Respir Cell Mol Biol Vol 54, Iss 5, pp 697-706, May 2016

Copyright $\odot 2016$ by the American Thoracic Society

Originally published in Press as DOI: 10.1165/rcmb.2014-0056OC on October 14, 2015

Internet address: www.atsjournals.org 
injury in mice (4), and this was accompanied by reduced levels of both basal and inducible expression of the proteins required for mitigating cellular stress (5). Recently, we have shown that targeted deletion of Nrf2 in airway epithelium results in cellular damage, exacerbates hyperoxic lung injury, and impairs the resolution of lung inflammation (6), demonstrating an important role for a lung epithelium-specific NRF2-driven transcriptional response in mitigating cellular stress induced by prooxidants.

Under physiological conditions, nuclear levels of NRF2 are maintained at basal level, because this transcription factor is localized mainly in the cytosol and is subject to Kelch-like ECH-associated protein 1 (KEAP1)-mediated ubiquitination and proteasomal degradation (7); however, in response to cellular stress, NRF2 escapes KEAP1mediated degradation and accumulates in the nucleus, where it transcriptionally induces the antioxidant response element (ARE)-mediated gene expression. NRF2 levels are also modulated by its acetylation/deacetylation and phosphorylation/dephosphorylation status, which appear to occur in an inducer-specific manner (5). For instance, NRF2 is phosphorylated by glycogen synthase kinase- $3 \beta$, and this modification prevents the entry of NRF2 into the nucleus, resulting in impaired AREmediated gene expression in human embryonic kidney $293 \mathrm{~T}$ cells in response to cellular stress (8). p300/cAMP response element-binding protein-binding protein-mediated NRF2 acetylation is required for its increased nuclear accumulation and ARE binding in mammary epithelial cells exposed to toxicants such as arsenite (9).

Among class III histone deacetylases, sirtuin 1 or silent information regulator 1 (SIRT1) has been implicated in the regulation of cell growth and survival (10-11). It regulates various cellular processes, including inflammation, chromatin stability, and oxidative stress, by deacetylating various proteins, including nuclear factor- $\kappa \mathrm{B}$, forkhead box protein O3 (FOXO3), and hypoxia-inducible factor $1 \alpha$ (12). Neither the stability nor the activity of NRF2 in BEAS-2B cells treated with $\mathrm{H}_{2} \mathrm{O}_{2}$ was found to be affected by SIRT1 inhibition (13). In contrast, a recent study in erythroid K562 cells has reported that ectopic SIRT1 causes NRF2 deacetylation and prevents its binding to DNA (14); however, the exact role of SIRT1 in the regulation of NRF2 activation and its target gene expression in lung epithelial cells during acute and chronic hyperoxic stress remain poorly understood. Thus, to determine whether crosstalk exists between SIRT1 and NRF2 in the regulation of hyperoxia-induced lung epithelial cell death, we used cell-based systems involving nonmalignant human small airway epithelial cells (HSAECs) and mouse lung type-II alveolar epithelial cells. Here, we report that SIRT1 promotes hyperoxic lung epithelial cell injury and death in vitro and in vivo, and this occurs through deregulated anti- and pro-apoptotic balance in chronic hyperoxia.

\section{Materials and Methods}

For additional details of methods, reagents, and primer sequences, see the online supplement.

\section{Cell Culture and Hyperoxia Exposure}

HSAEC line immortalized with human telomerase reverse transcriptase was grown in Dulbecco's modified Eagle medium with Ham's F12 nutrient mixture (DMEM/F12) in the presence of $10 \%$ fetal bovine serum (15). Cells were plated in equal number, grown to $70-80 \%$ confluence, and then placed in the exposure chamber (BillupsRothenberg, Del Mar, CA). The chamber was filled with a gas mixture containing $95 \% \mathrm{O}_{2}$ and $5 \% \mathrm{CO}_{2}$ and was refilled every 12 hours thereafter. As a control group, cells were placed in the regular cell culture incubator. Culture medium was changed every 24 hours during the exposure period. For inhibitor/activator studies, cells were pretreated with vehicle (dimethyl sulfoxide [DMSO]), sirtinol (10 $\mu \mathrm{M}$; Sigma, Cat No. S7942), or resveratrol (10 $\mu \mathrm{M}$; Sigma, Cat. No. R5010) for 6 hours in serum-free medium and were exposed to hyperoxia or room air in complete medium. For in vivo animal studies, see the online supplement.

\section{Cell Viability Assays}

Cells in equal number were plated and exposed to hyperoxia as described above. Cell viability was quantified by

3-(4,5-dimethylthiazol-2-yl)-2,5-

diphenyltetrazolium bromide (MTT) assay.
LDH release was measured by a CytoTox 96 Non-Radio Cytotoxicity assay kit (Promega, Madison, WI). Viability and lactate dehydrogenase $(\mathrm{LDH})$ release were calculated as a percentage of increase or decrease over their respective room air controls.

\section{Transfections and Reporter Gene Analyses}

Cells were transfected with the $\mathrm{NAD}^{+}$ phosphate reduced: quinone oxidase reductase-1 (NQO1, kindly provided by Jeffery Johnson) (16) or Heme oxygenase 1 (HMOX1, kindly provided by Jawed Alam) promoter reporter (luciferase [Luc]) construct (17) (100 ng), along with the Renilla Luc plasmid, pRL-TK (5 ng; Promega Corp., Madison, WI). At 24 hours after transfection, cells were treated with either DMSO or sirtinol and exposed to hyperoxia for different time points as indicated. Extracts were assayed for firefly and the Renilla Luc activities using a dual Luc kit (Promega Corp.). Firefly Luc activity was normalized to that of Renilla.

\section{Gene Expression Analysis}

Quantitative reverse transcriptasepolymerase chain reaction was performed by SYBR-green-based assays, and immunoblot analysis was performed using indicated antibodies by standard methods.

\section{Small Interfering RNA Transfection} siGENOME Smart pool small interfering RNAs (siRNAs) specific for NRF2 or NFE2L2 (M-003755-02), SIRT1 (M-003540-01), and nontargeting scrambled (Scr) siRNA (pool 2; D-001206-14-05) were obtained from Dharmacon (Lafayette, CO). Cells were transfected with siRNA (20 nM) using DharmaFECT1 reagent and at 24 hours after transfection were exposed to either room air or hyperoxia.

\section{Nuclear Extracts Preparation}

Nuclear protein was prepared by using the NI-PER kit (Thermo Scientific, Waltham, MA). Nuclear protein was separated on a $10 \%$ sodium dodecyl sulfate-polyacrylamide gel electrophoresis, and the membranes were probed with NRF2 and Lamin A/C antibodies (Santa Cruz Biotechnology Inc., Dallas, TX). 


\section{Immunoprecipitation and Immunoblotting}

Cells were exposed to hyperoxia and lysed in immune precipitation assay buffer, and cell lysates $(500 \mu \mathrm{g})$ were precleared with Protein A/G-agarose beads, and centrifuged $(1,000 \times g$ for $2 \mathrm{~min})$. Supernatant was incubated with antiacetylated lysine antibodies $(2 \mu \mathrm{g})$ for 4 hours at $4^{\circ} \mathrm{C}$, and the immune complexes were precipitated with Protein A-agarose beads. The immunoprecipitates were separated on a sodium dodecyl sulfate-polyacrylamide gel electrophoresis and were probed with anti-NRF2 antibody.

\section{Statistical Analyses}

Data were expressed as mean \pm SEM. A $P$ value $\leqslant 0.05$ was considered significant and was determined using the Student's $t$ test.

\section{Results}

\section{SIRT1 Inhibition Prevents Hyperoxia-Induced Lung Epithelial Death without Augmenting NRF2-Dependent Antioxidant Enzyme Expression}

To elucidate the role of SIRT1 in the regulation of hyperoxic cell death, we pretreated HSAECs with SIRT1 inhibitor (sirtinol) or vehicle (DMSO) and then exposed them to hyperoxia for 36 hours. As shown in Figure 1A, the number of live cells in the sirtinol-treated group was greater than in the vehicle-treated group when observed under the microscope. To further verify this result, we used MTT and LDH assays to quantify the cell viability and toxicity, respectively (Figure 1B). MTT assays showed that $\sim 47 \%$ of the sirtinol-treated cells were viable, as compared with only $39 \%$ of the cells in the vehicle-treated group

(Figure 1B). Consistent with these results, we detected lower levels of LDH release in the sirtinol-treated cells than in their vehicle-treated counterparts (right panel). Sirtinol also conferred protection against hyperoxia in a mouse type 2-like alveolar epithelial cell line (see Figure E1 in the online supplement). SIRT1 activator, resveratrol, enhanced cell death and toxicity in both HSAECs and mouse type 2-like alveolar epithelial cells (Figure E2).

To determine whether SIRT1 promotes cell death by dampening NRF2-regulated

A

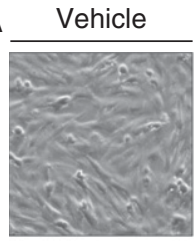

Sirtinol
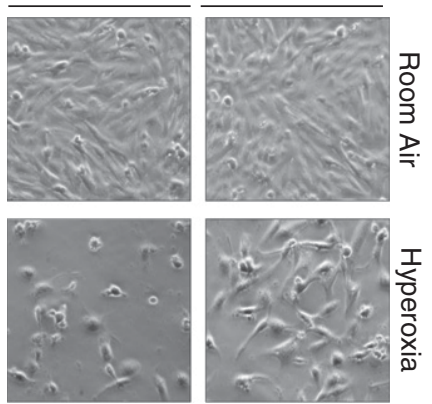

HMOX1-ARE-LUC
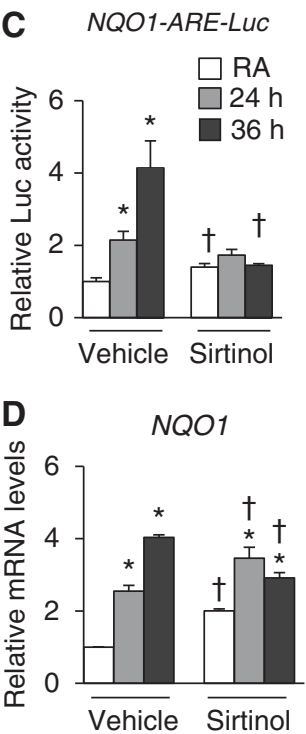
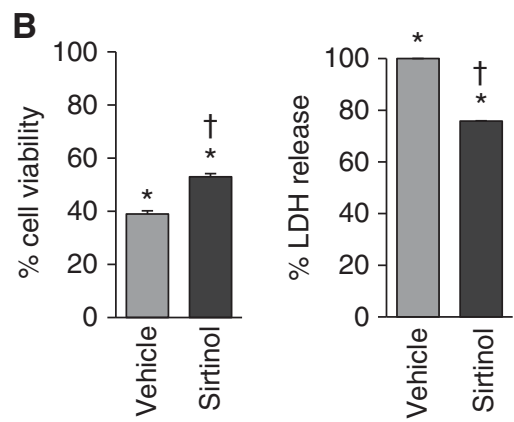

Figure 1. The effects of sirtinol on chronic hyperoxia-induced cell death and antioxidant enzyme (AOE) expression. After 6-hour pretreatment with sirtinol or vehicle, human small airway epithelial cells (HSAECs) were exposed to hyperoxia for 24 or 36 hours or to room air, and cell viability was measured. (A) Photomicrographs of cells after exposure to 36 hours hyperoxia or room air. $(B)$ Quantification of cell viability by 3-(4,5-dimethylthiazol-2-yl)-2,5-diphenyltetrazolium bromide (MTT) and lactate dehydrogenase (LDH) assays after 36 hours hyperoxia. LDH levels from vehicle-treated hyperoxia-exposed samples are considered as 100\%. (C) NAD ${ }^{+}$phosphate reduced: quinone oxidase reductase-1 (NQO1) and Heme oxygenase 1 (HMOX1) promoter activity after 24 and 36 hours of hyperoxia. ( $D$ and $E$ ) Nuclear factor (erythroid-derived 2)-like 2 (NRF2)-dependent AOE expression was analyzed by quantitative RT-PCR (qRT-PCR). Data are presented as mean \pm SEM $(n=3-4)$. Values from the vehicle-treated room air group are considered as 1 or $100 \%$. ${ }^{\star} P \leqslant 0.05$ room air versus hyperoxia; ${ }^{\dagger} P \leqslant 0.05$, vehicle versus sirtinol. ARE, antioxidant response element; GCLC, glutamate-cysteine ligase, catalytic subunit; GCLM, glutamate-cysteine ligase, modifier subunit; Luc, luciferase; RA, room air.

antioxidant enzyme (AOE) expression, we transfected cells with a NQO1-Luc or HMOX1-Luc reporter construct bearing the NRF2 binding site, ARE. These cells were treated with sirtinol or vehicle and were then exposed to hyperoxia for 24 or 36 hours. Both the NQO1-Luc and the HMOX1-Luc activities in sirtinol-treated cells were significantly higher under basal (room air-exposed) conditions (Figure 1C), but their overall induction (relative-fold increase) was significantly lower under hyperoxic conditions than that of their vehicle-treated counterparts. We found increased levels of NQO1 (2-fold) and HMOX1 ( 2.5-fold) mRNA in sirtinol-treated cells under basal conditions, which was consistent with these results, but their induction levels were lower under hyperoxic conditions (Figure 1D). We also examined the expression status of glutamate-cysteine ligase, modifier subunit (GCLM) and glutamate-cysteine ligase, catalytic subunit (GCLC), which are 
well-established target genes of NRF2. We found that GCLM and GCLC levels were elevated in cells treated with sirtinol under basal conditions (Figure 1E); however, the relative mRNA levels of GCLM and GCLC were lower in sirtinol-treated cells exposed to 36 hours of hyperoxia (Figure 1E).

\section{SIRT1 Inhibition Augments AOE Expression in Response to Acute Hyperoxia}

We next determined whether SIRT1 differentially modulates NRF2-dependent AOEs in response to acute (6-12 h) hyperoxia. This exposure did not cause
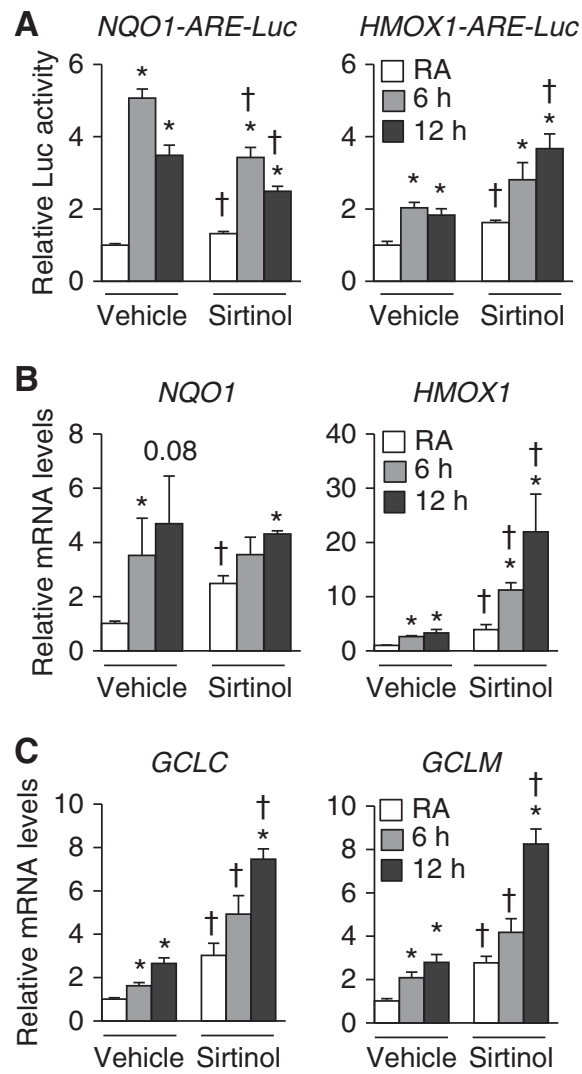

Figure 2. Nrf2-dependent AOE expression in sirtinol-treated cells exposed to acute hyperoxia. HSAECs were pretreated with sirtinol for 6 hours and exposed to room air or hyperoxia for 6 to 12 hours. (A) NQO1-ARE and HMOX1-ARE luciferase activity after 6 and 12 hours hyperoxia. (B) NQO1 and HMOX1 and (C) GCLC and GCLM mRNA expressions were analyzed by qRT-PCR. Data are presented as mean \pm SEM $(n=3-4)$. Values from the vehicle-treated room air-exposed cells are considered as one unit. ${ }^{*} P \leqslant 0.05$, room air versus hyperoxia; ${ }^{\dagger} P \leqslant 0.05$, vehicle versus sirtinol. a detectable level of cell death and toxicity when compared with room air-exposed cells (data not shown). As anticipated, based on the data in Figure 1, SIRT1/2 inhibition modestly increased both NQO1-Luc and HMOX1-Luc activities under basal conditions (Figure 2A); however, hyperoxia (6 or $12 \mathrm{~h}$ )-induced NQO1-Luc reporter activity was significantly lower than in their vehicle-treated counterparts, and HMOX1-Luc activity was higher. In agreement with this increased basal level of promoter activity, sirtinol increased both NQO1 and HMOX1 mRNA (Figure 2B) and protein (Figure E3) expression under basal conditions. However, the relative mRNA levels of NQO1 were lower and those of HMOX1 were higher in the sirtinol-treated cells exposed to hyperoxia for 6 or 12 hours. The relative mRNA levels of NQO1 and HMOX1 under basal and acute hyperoxic conditions corresponded to the respective ARE-driven promoter activities. In addition, we found that GCLM and GCLC levels were elevated in cells treated with sirtinol under basal and hyperoxic conditions (Figure 2C).

Together, these results suggest that SIRT1 dampens NRF2-regulated AOE expression under both basal and acute hyperoxic conditions, but it promotes their expression in chronic hyperoxia. In agreement with this result, increased expression of HMOX1 NQO1, GCLM, and GCLC was observed in resveratrol-treated and hyperoxia-exposed HSAECs compared with their vehicle-treated counterparts (Figure E4). Resveratrol had no significant effect on the NRF2 target gene (except HMOX1) expression that was induced by acute hyperoxia (Figure E4).

\section{SIRT1 Depletion Attenuates Hyperoxic Cell Death and AOE Expression}

Because sirtinol inhibits both SIRT1 and SIRT2, we examined the specific contribution of SIRT1 to hyperoxia-induced cell death and NRF2-regulated AOE expression by using an siRNA-mediated knockdown approach. SIRT1 mRNA expression was significantly induced by hyperoxia at both 24 and 36 hours in control Scr siRNA-transfected cells (Figure 3A), but its expression was markedly lower in room air- and hyperoxia-exposed cells transfected with SIRT1 siRNA. Knockdown of SIRT1 was verified by immunoblot analysis (Figure 3B). The SIRT1 siRNA-transfected cells showed greater resistance to hyperoxia than did their Scr siRNA-transfected counterparts (Figures $3 \mathrm{C}$ and $3 \mathrm{D}$ ), suggesting that SIRT1 mediates hyperoxic cell death. It should be noted that because the $\mathrm{LDH}$ release results corresponded closely to the results of the MTT assay (Figure 1B), we chose to determine cell death by measuring LDH levels in culture supernatants from this point forward, using the cells for RNA and protein isolation. To determine whether SIRT1 promotes cell death by dampening NRF2 signaling, we analyzed the expression of AOEs in Scr siRNA- and SIRT1 siRNA-transfected cells exposed to room air and chronic hyperoxia. As shown in Figure 3E, NQO1, HMOX1, and GCLM mRNA levels were lower in SIRT1 siRNA-transfected cells exposed to 24 or 36 hours hyperoxia than in their Scr siRNA-transfected counterparts. We also determined the effects of SIRT1 depletion on NRF2 target gene expression in response to acute hyperoxia. As shown in Figure 3F, we found significantly reduced levels of NRF2-regulated antioxidant gene induction in SIRT1depleted cells exposed to 6 hours hyperoxia but not in the 12-hour hyperoxia condition. Unlike sirtinol treatment, siRNA-mediated SIRT1 depletion did not significantly affect the basal-level expression of AOEs.

\section{NRF2 Depletion Augments Hyperoxia- Induced Cell Death and Diminishes AOE Expression}

To ascertain the protective functions of NRF2 in hyperoxia, we transfected cells with NRF2 siRNA or Scr siRNA and then exposed them to hyperoxia. We found that NRF2 mRNA expression was significantly lowered in hyperoxia-exposed NRF2 siRNA-transfected cells (Figure 4A). This knockdown of NRF2 mRNA expression was verified by immunoblot analysis (Figure 4B). Unlike SIRT1 siRNA-mediated knockdown, which resulted in more than $80 \%$ knockdown efficiency, we noted about $50 \%$ depletion of the endogenous NRF2 mRNA and protein levels in the NRF2 siRNA-transfected cells. Nevertheless, NRF2 depletion resulted in increased hyperoxic death when compared with similarly treated Scr siRNA-transfected 
A

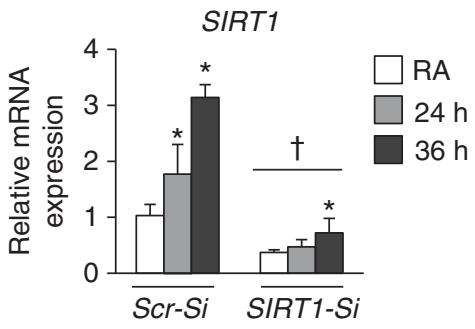

C

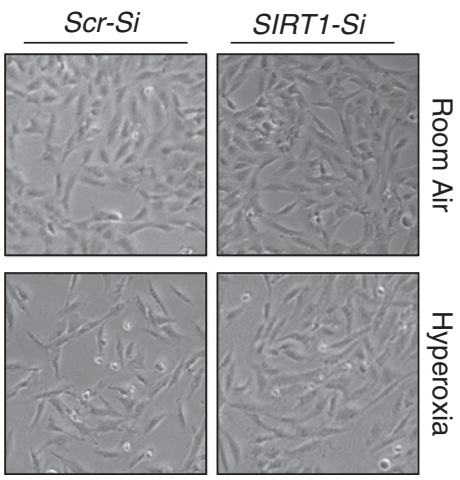

E

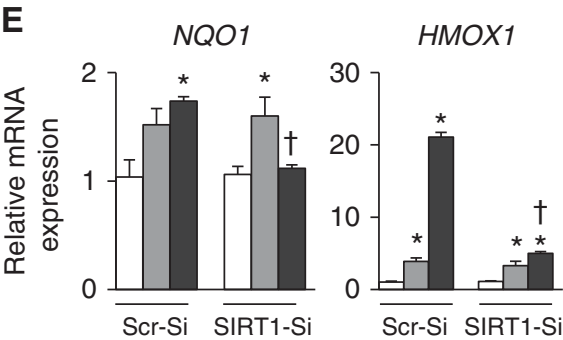

$\mathbf{F}$

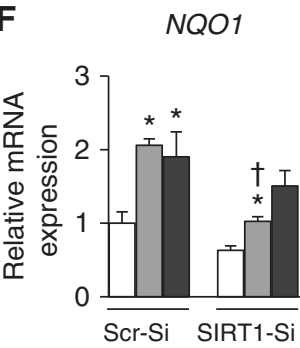

B

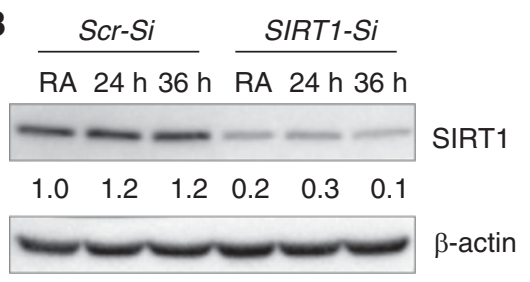

in Figure 4E, NRF2 depletion markedly reduced both basal and hyperoxia-induced expression of NQO1 and GCLM mRNA, but the levels of GCLC were modestly affected, and HMOX1 was not altered by the NRF2 deficiency. As we only depleted $\sim 50 \%$ of the endogenous NRF2, the induction of HMOX1 expression in the NRF2 siRNA-transfected cells could be attributable to the residual NRF2. Alternatively, other transcription factors are able to compensate NRF2 in regulating the activation of HMOX1 promoter, which contain multiple cis-acting elements, such as activator protein 1 (AP1).

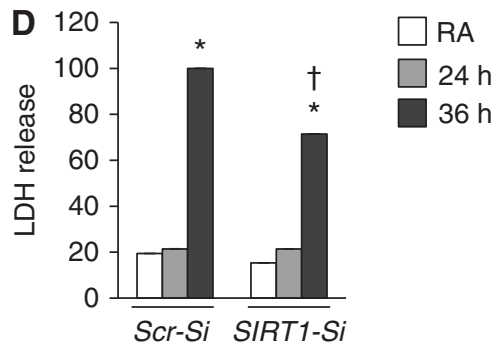

\section{SIRT1 Activation Augments Hyperoxia-Induced Cell Death in NRF2-Deficient Cells}

To determine whether SIRT1 promotes hyperoxic cell death in an NRF2dependent or -independent manner, we depleted NRF2 in HSAECs by an siRNA-mediated approach and then exposed them to hyperoxia in the presence or absence of resveratrol (SIRT1 activator) for 36 hours. MTT and LDH assays were used to quantify cell viability and toxicity, respectively (Figure 4F). MTT assays showed that $\sim 21 \%$ of the resveratrol-treated or NRF2 siRNA-transfected cells were viable, as compared with vehicle-treated or Scr siRNA-transfected cells, which showed 34\% viability (Figure 4F, left panel); however, the viability was significantly lower (11.7\%) in NRF2 siRNA-transfected/ resveratrol-treated cells. Accordingly, LDH release in resveratrol-treated or NRF2 siRNA-transfected cells exposed to hyperoxia was higher than in their control groups (Figure $4 \mathrm{~F}$, right panel). LDH release was greater in NRF2 siRNA-transfected/resveratrol-treated cells than in Scr siRNA-transfected/ resveratrol-treated cells. These results suggest that SIRT1 promotes hyperoxiainduced cell death independent of NRF2.

\section{SIRT1 Inhibition Reduces Hyperoxia-Induced Death Effector Activation}

The data presented above demonstrate that the inhibition or siRNA-mediated depletion of SIRT1 attenuates hyperoxic death, but NRF2 knockdown augments it. Contrary to our expectation, NRF2-regulated AOE induction was

cells (Figures 4C and 4D). NRF2 depletion also enhanced the susceptibility of the lung epithelial cells to hyperoxia, because significant death was detectable at the 24-hour time point in cells transfected with NRF2 siRNA, but not with Scr siRNA. We next determined the effects of NRF2 deficiency on AOE expression. As shown 
A

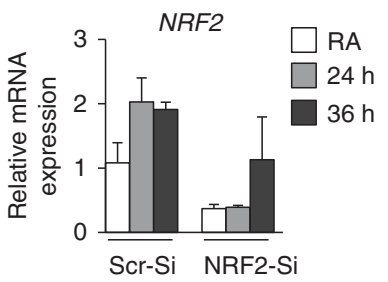

C

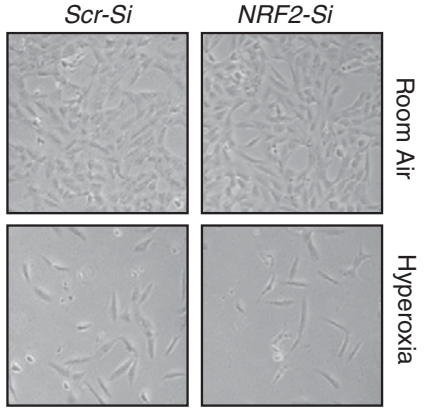

E

$\mathbf{F}$

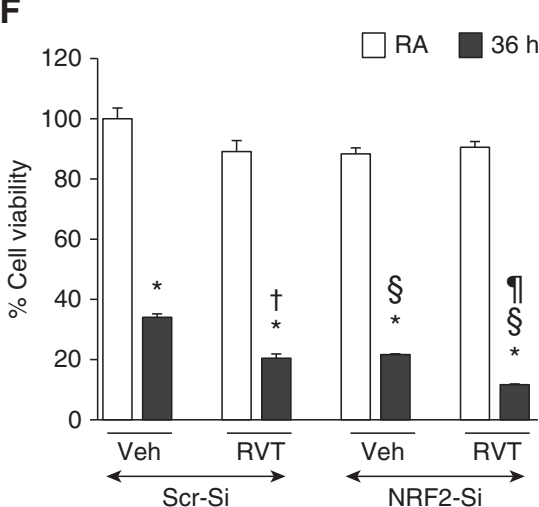

B

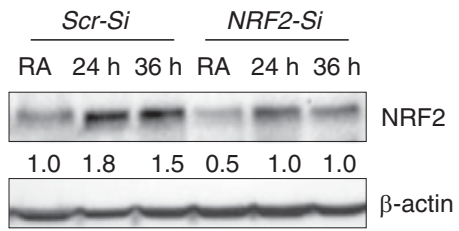

D

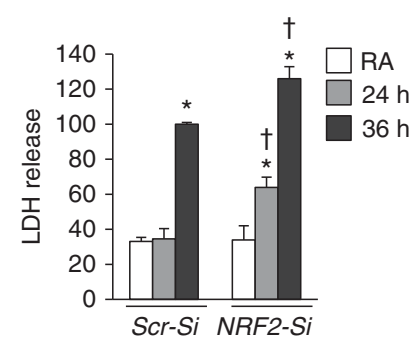

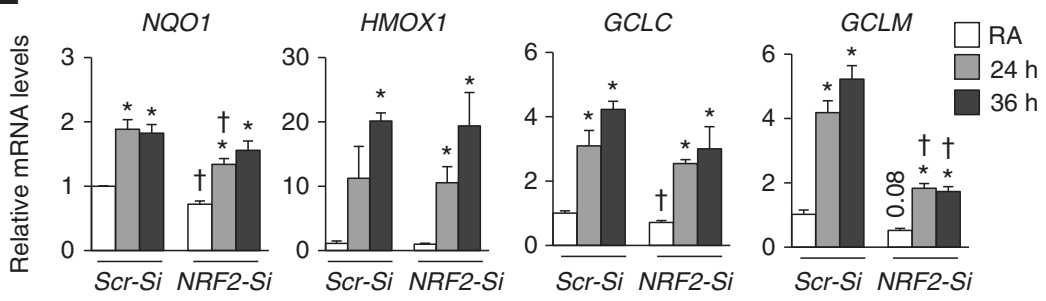

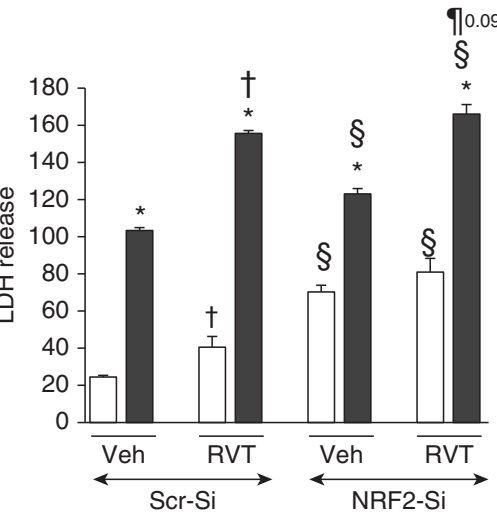

Figure 4. NRF2 depletion increases hyperoxic cell death, and SIRT1 activation further augments it. (A) qRT-PCR analysis of NRF2 mRNA expression in HSAECs transfected with either Scr siRNA or NRF2 siRNA. Expression of NRF2 was calculated relative to Scr siRNA-transfected room air-exposed samples. (B) Immunoblot analysis of NRF2 in Scr siRNA- or NRF2 siRNA-transfected cells exposed to room air or hyperoxia. Relative NRF2 band intensity was calculated using $\beta$-actin as a reference. $(C)$ Photomicrographs of cells after exposure to 36 hours of hyperoxia or room air. (D) Quantification of LDH levels. (E) AOE expression analyzed by qRT-PCR. Data are presented as mean \pm SEM $(n=3-4)$. Values from the Scr siRNA-transfected room air-exposed samples are considered as one. ${ }^{\star} P \leqslant 0.05$, room air versus hyperoxia; ${ }^{\dagger} P \leqslant 0.05$, Scr siRNA versus NRF2 siRNA. $(F)$ The effect of RVT on chronic hyperoxia-induced death in Nrf2-deficient HSAECs. Cells were transfected with Scr or NRF2 siRNA, then pretreated with RVT (10 $\mu \mathrm{M})$ or Veh for 6 hours and exposed to hyperoxia or room air. Quantification of cell viability evaluated by MTT and LDH assays after 36 hours hyperoxia. Cell viability from Veh-treated hyperoxia-exposed Scr siRNA-transfected cells are considered as $100 \%$. Data are presented as mean \pm SEM $(n=4)$. ${ }^{\star} P \leqslant 0.05$, room air versus hyperoxia; ${ }^{\dagger} P \leqslant 0.05$, Veh versus RVT; ${ }^{\S} P \leqslant 0.05$, Scr siRNA versus NRF2 siRNA or RVT; "Scr siRNA-transfected RVT-treated versus NRF2 siRNA-transfected RVT-treated cells. RVT, resveratrol; Veh, vehicle.

markedly lower in either sirtinol-treated or SIRT1-depleted cells exposed to chronic hyperoxia. SIRT1 activation further augments hyperoxic death in cells with NRF2-deficiency, suggesting that SIRT1 promotes cell death through a different mechanism. An imbalance between pro-apoptotic BCL2-associated agonist of cell death (BAD) and BCL2-associated X protein 1 (BAX1) and anti-apoptotic B-cell lymphoma 2 (BCL2) and BCL2-like 1 isoform 1 (BCLXL) proteins plays a key role in hyperoxic cell death (18). Thus, to determine the relevance of the anti-/pro-apoptotic imbalance to the regulation of hyperoxiainduced cell death, we treated the cells with the pan caspase inhibitor, $z$-VAD-fmk, prior to exposing them to hyperoxia and measuring cell death. As indicated in Figure 5A, z-VAD-fmk markedly attenuated hyperoxic cell death. We analyzed the effects of SIRT1 inhibition/depletion and NRF2 depletion on the expression levels of pro-apoptotic (BAD and BAX1) and anti-apoptotic (BCL2 and BCLXL) genes. Pro-apoptotic (BAD and BAX1) and anti-apoptotic (BCL2 and BCLXL) gene expressions were not affected in acute hyperoxia, but they were differentially (up and down) induced by SIRT1 and NRF2 deficiency in the setting of chronic hyperoxia (Figure E5) and acute hyperoxia (Figure E6); thus, we measured the levels of proteolytic cleaved products of caspase 3 and/or poly(ADP-ribose) polymerase 1 (PARP-1) as the final readout of antiand pro-apoptotic imbalance, by immunoblot analysis (Figure 5B). As expected, hyperoxia activated caspase 3 and PARP-1, but their cleavage levels were lower in sirtinol-treated cells. In addition, we found increased expression levels of BCL2, an anti-apoptotic protein, in sirtinol-treated cells exposed to chronic hyperoxia compared with vehicle-treated cells, and this was associated with reduced levels of cleaved caspase 3 (Figure 5C).

To ascertain the role of SIRT1 in this process, we measured cleaved caspase 3 and BCL2 levels in SIRT1-depleted cells exposed to hyperoxia and found that they were lower than in the corresponding Scr siRNA-treated control cells (Figure 5D). These results are consistent with the reduced levels of hyperoxic cell death 
A

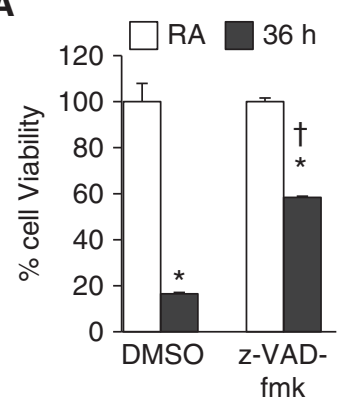

B

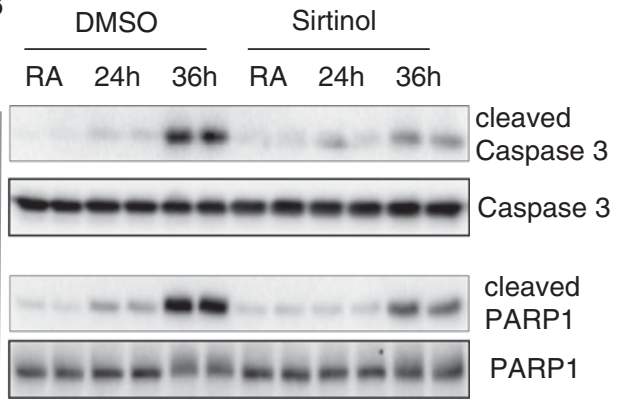

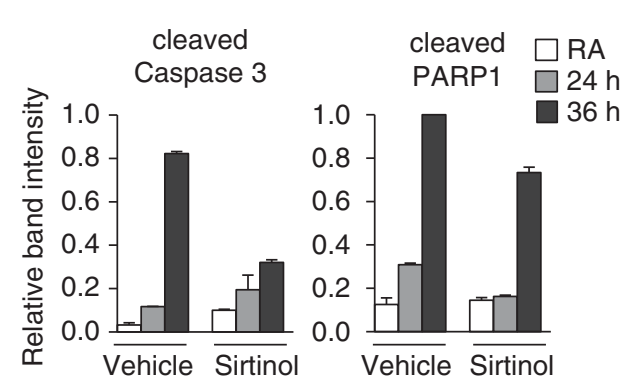

D
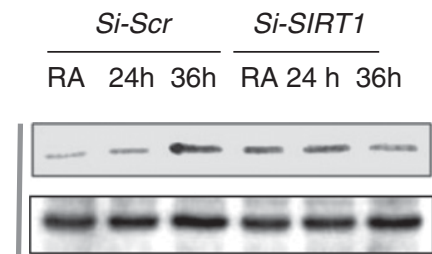
Casapse 3 Caspase 3

C

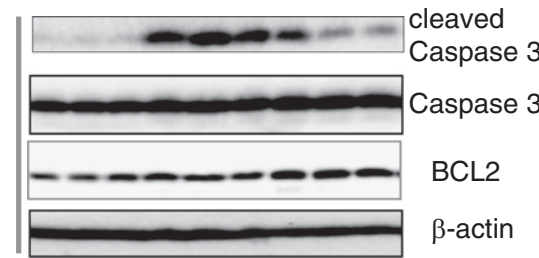

RA 24h 36h RA 24 h $36 \mathrm{~h}$

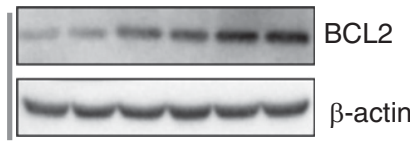

Figure 5. SIRT1 promotes hyperoxia-induced caspase 3 activation. (A) The effects of z-VAD-fmk on hyperoxia-induced cell death. HSAECs were exposed to hyperoxia in the presence of $z$-VAD-fmk $(10 \mu \mathrm{M})$, and cell viability was measured by MTT assay. ${ }^{*} P<0.05$, room air versus hyperoxia; ${ }^{\dagger} P<0.05$, DMSO versus z-VAD-fmk. (B) Immunoblot analysis of hyperoxia-induced caspase 3 and poly(ADP-ribose) polymerase 1 (PARP-1) activation (left panel) in cells treated with vehicle (DMSO) or sirtinol. Right panel, quantification cleaved caspase 3 and PARP-1 levels. (C) Immunoblot analysis of caspase 3 and BCL2 expression in cells treated with vehicle or sirtinol and exposed to room air and hyperoxia. (D) Immunoblot analysis of cleaved caspase 3 (left panel) and BCL2 (right panel) in Scr siRNA- or SIRT1 siRNA-transfected cells exposed to room air and hyperoxia. BCL, B-cell lymphoma; z-VAD-fmk, caspase inhibitor.

detected in SIRT1-inhibited or -depleted cells.

\section{SIRT Inhibition Increases Nuclear NRF2}

To determine whether SIRT1 inhibition affects NRF2 levels, lysates were isolated from HSAECs exposed to hyperoxia for 24 or 36 hours in the presence or absence of sirtinol and were subjected to Western blot analysis using NRF2 antibodies (Figure 6A). This analysis revealed increased levels of NRF2 following hyperoxia in vehicle-treated cells, but the levels were elevated under basal and lower in hyperoxic conditions in sirtinol-treated cells. Nuclear accumulation of NRF2 is associated largely with enhanced ARE-mediated expression of AOEs. Therefore, to determine whether SIRT1 inhibition increases nuclear NRF2 levels, nuclear extracts were prepared from these cells, and NRF2 levels were analyzed by immunoblotting (Figure 6B). In agreement with the Western blot data, we found increased levels of nuclear NRF2 in cells exposed to hyperoxia for 24 hours (lane 2) or 36 hours (lane 3) when compared with control cells exposed to room air (lane 1). We found higher levels of nuclear NRF2 in sirtinol-treated cells under basal (lane 4) and 36-hour hyperoxic (lane 6) conditions than in their vehicle-treated counterparts; however, this result at the 36-hour time point did not corroborate with the Western blot analysis of total cell lysates, which showed decreased levels of NRF2 in hyperoxic condition. It is unclear whether this discrepancy is attributable to differences between whole cell lysate and nuclear extract procedures or an altered availability of NRF2 epitopes. Immunoblot analysis of cytoplasmic and nuclear compartments revealed very low levels of NRF2 under basal conditions, whereas hyperoxia stimulated its nuclear accumulation (Figure E7).

\section{Hyperoxia Induces NRF2 Acetylation in Acute Hyperoxia but Not in Chronic Hyperoxia}

We next determined whether hyperoxia modulates NRF2 acetylation levels and, if so, whether this modulation is mediated in a SIRT1-dependent manner. Cells were exposed to room air or hyperoxia in the absence and presence of sirtinol, and cellular extracts were prepared and immunoprecipitated with antiacetylated lysine antibodies. The samples were subjected to Western blot analysis with anti-NRF2 antibodies. As shown in Figure 6C, the levels of acetylated NRF2 in cells exposed to hyperoxia for 24 hours (lane 2) or 36 hours (lane 3) were not greater than those in the room-air control cells (lane 1). Moreover, sirtinol did not alter the levels of acetylated NRF2 under either basal (lane 4) or hyperoxic (lanes 5 and 6) conditions (Figure 6C). Immunoblots developed under prolonged exposure did not reveal differences in the acetylated NRF2 levels between hyperoxia and room air-exposed samples when compared with IgG control cells (Figure E8). In contrast, the levels of acetylated NRF2 in cells exposed to hyperoxia for 6 hours (lane 2) were strikingly higher than in cells exposed to room-air (lane 1) and 12-hour hyperoxia, and sirtinol blocked NRF2 acetylation induced by hyperoxia (lane 5 versus lane 2). It should be noted that NRF2 acetylation levels did not correspond with increased levels of NRF2. 


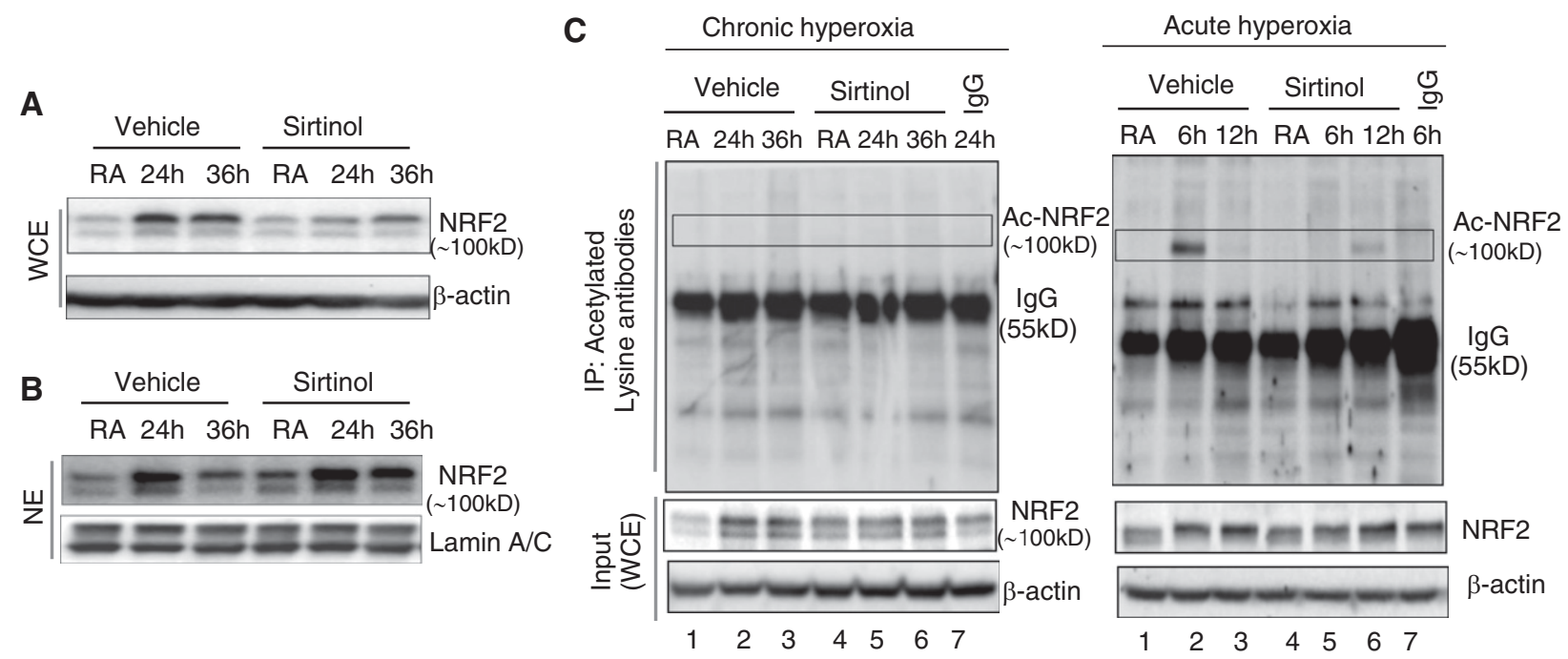

Figure 6. NRF2 nuclear accumulation and acetylation in hyperoxia-exposed cells with SIRT1 inhibition. HSAECs pretreated with sirtinol or vehicle were exposed to hyperoxia or RA, WCE $(A)$, or nuclear extracts (NEs) $(B)$ prepared and immunoblotted with anti-NRF2, $\beta$-actin, or lamin A/C antibodies. (C) HSAECs pretreated with sirtinol or vehicle were exposed to chronic (24 or $36 \mathrm{~h}$ ) (left panel) or acute (6 or 12 h) (right panel) hyperoxia or RA, lysates prepared, and $500 \mu \mathrm{g}$ lysates pooled from three independent biological replicates $(n=3)$ were immunoprecipitated (IPed) with antiacetylated lysine antibodies. IPed samples were immunoblotted with the anti-NRF2 antibodies. In parallel, whole cell lysates (40 $\mu$ g) were separated and immunoblotted with anti-NRF2 and $\beta$-actin antibodies (lower panel). Note that the images of the immunoblots developed for 60 seconds of exposure are presented. Images of a longer (10-min) exposure are shown in Figure E7. Ac, acetylation; IP, immunoprecipitation; WCE, whole cell extracts.

\section{SIRT1 Inhibition Attenuates}

\section{Hyperoxia-Induced Acute Lung Injury}

We next determined the expression levels of Sirt1 in the lungs of mice exposed to hyperoxia. As shown in Figure 7A, we found elevated levels of Sirt1 expression in the lungs of mice exposed to hyperoxia for 48 hours or 60 hours. To examine the relevance of Sirt1 in mediating hyperoxia-induced lung injury, mice treated with sirtinol or vehicle were exposed to hyperoxia for 60 hours, and bronchoalveolar lavage (BAL) fluids were collected. Protein concentration and $\mathrm{LDH}$ release in the BAL fluid were measured as a read-out of lung alveolar epithelial injury (Figure 7B). We found reduced levels of hyperoxia-induced alveolar permeability (BAL protein) and toxicity (LDH levels) in the lungs of sirtinol-treated mice than in those of their vehicle-treated counterparts, suggesting that Sirt1 promotes hyperoxia-induced lung injury.

\section{Discussion}

Persistent cellular damage and death of lung epithelial cells after acute lung injury are associated with abnormal tissue repair and inflammation, and dysfunctional NRF2 signaling has been implicated in these pathologies (4). SIRT1 regulates the gene expression involved in cell growth, survival, and metabolism in response to chronic physiological and pathological stresses, primarily by deacetylating several transcription factors, signaling molecules, and chromatin-bound histones (12). Using pharmacological inhibition and siRNA-mediated depletion approaches, we have demonstrated that SIRT1 is a critical positive regulator of hyperoxia-induced lung epithelial cell death and that it mediates this effect by altering the balance between pro-apoptotic and anti-apoptotic genes, not by attenuating NRF2-regulated AOE transcription in chronic hyperoxia. Oxidative stress primarily contributes to the activation of death effector pathways in lung epithelial cells in response to hyperoxic insult (19-23). The results obtained in our current study are counterintuitive in that, despite the lack of a greater induction of AOEs in chronic hyperoxia, sirtinol-treated or SIRT1-depleted lung epithelial cells showed decreased susceptibility to hyperoxia. We recently reported that NRF2 activation and NRF2-dependent AOE expression in lung epithelial cells are not impaired largely in chronic hyperoxia (17). NRF2 depletion, however, enhanced susceptibility to hyperoxia (Figure 4), suggesting that this transcription factor exerts a prosurvival response during chronic hyperoxia by inducing antioxidant gene expression. We noted that SIRT1-depleted cells are less susceptible to hyperoxia despite their reduced expression of NRF2-regulated antioxidant genes (NQO1, GCLC, and GCLM) (Figure 1). In addition, we noted that resveratrol (SIRT1 activator) further augments hyperoxia-induced death in cells with NRF2 knockdown (Figure 7A); thus, it is likely that SIRT1 promotes cell death by triggering the activation of death effector signaling in chronic hyperoxia, independent of NRF2-regulated AOE expression status.

Deregulated expression and/or activation of the BCL2 family of pro-apoptotic (such as BAD/BAX) and anti-apoptotic (BCL2/BCLXL) proteins are essential for triggering oxidant-induced apoptosis (reviewed in Ref. 18). For example, overexpression of BCL2 or BCLXL in lung epithelial cells confers protection from hyperoxic death, whereas cells or mice lacking both Bax and Bak (BCL2-antagonist/ killer 1) are less susceptible to hyperoxia $(19,24)$. Indeed, we found a modest increase in the expression levels of BAD and BAX in cells exposed to chronic hyperoxia, but their induction was attenuated in both SIRT1-inhibited and SIRT1-depleted cells, suggesting that this deacetylase promotes cell death in part by up-regulating pro-apoptotic genes. Because apoptosis is a complex process involving simultaneous activation 

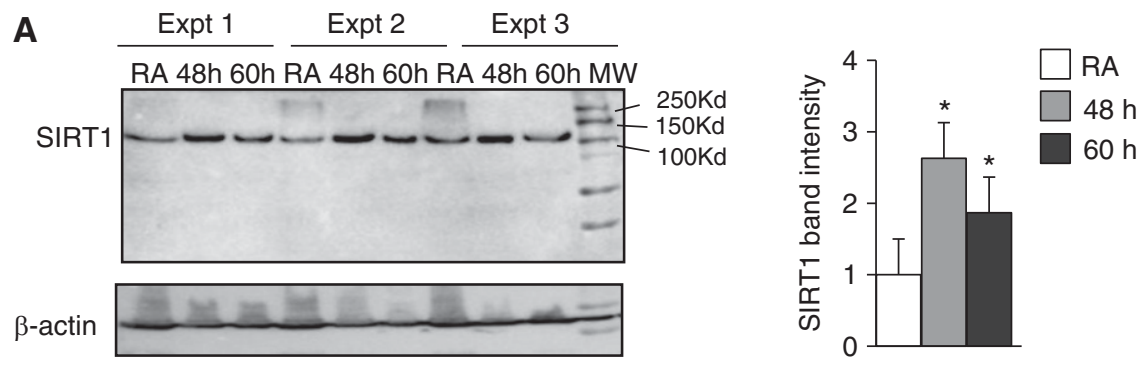

B
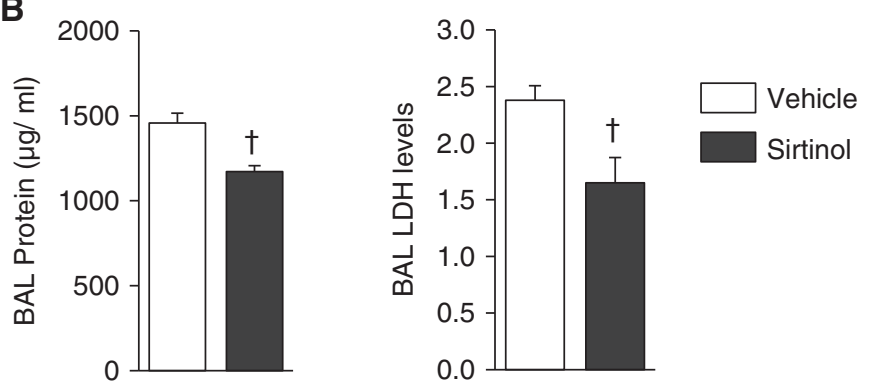

Figure 7. The effects of sirtuin (Sirt) inhibition on hyperoxia-induced lung injury. (A) Mice were exposed to RA or hyperoxia for 48 and 60 hours, lungs were harvested, and extracts were used for Western blot analysis using Sirt1 antibodies. Relative band intensity of Sirt1 was calculated using $\beta$-actin as a loading control, and values of RA samples were considered as one. Data are presented as mean \pm SEM. ${ }^{\star} P \leqslant 0.05$, RA versus hyperoxia. (B) Mice were administered sirtinol $(125 \mu \mathrm{g})$ or vehicle in $100 \mu \mathrm{L}$ volume intraperitoneally prior to hyperoxia exposure. Mice ( $n=3 /$ group) were given an additional dose after 24 hours of hyperoxia exposure. Mice were exposed to hyperoxia for 60 hours and killed.

Bronchoalveolar lavage (BAL) fluids were collected, and protein concentration and LDH levels were measured. Data are presented as mean \pm SEM. ${ }^{\dagger} P \leqslant 0.05$, vehicle versus sirtinol. Expt, experiment.

and inactivation of several pro- and antiapoptotic proteins, we analyzed the activation status of caspase 3 as a measure of pro-and anti-apoptotic imbalance during hyperoxic death in our experimental settings. We found reduced levels of caspase 3 activation in sirtinol-treated or SIRT1-depleted cells exposed to hyperoxia (Figure 5), further supporting our contention that SIRT1 promotes cell death by altering the balance between pro- and anti-apoptotic proteins leading to death effector activation. Although SIRT1 generally promotes cell survival and extends lifespan under stressful conditions, several studies have reported that it also activates cell death in response to oxidative stress. For example, inhibition of SIRT1 caused increased resistance to oxidant $\left(\mathrm{H}_{2} \mathrm{O}_{2}\right)$-induced stress in neurons (25), and its activation by resveratrol results in apoptosis of 3T3-L1 preadipocytes (26), suggesting that SIRT1 regulates cell survival in a contextual manner.

SIRT1 shuttles between the cytoplasmic and nuclear compartments and regulates gene expression in response to physiological and pathological cellular stresses (10-11). SIRT1 promotes cell survival under stressful conditions by deacetylating and inactivating various proteins (12) such as PARP-1 (27) and by up-regulating

FOXO3-dependent gene expression of p21 and p53 (28) and superoxide dismutase 2 gene expression (29). Nuclear accumulation of NRF2 generally leads to the up-regulation of several AOEs (7). Protein modifications of NRF2, after release from KEAP1-mediated retention, have been shown to influence the biology of NRF2. For example, it has been reported that p300/cAMP response elementbinding protein-binding protein-mediated NRF2 acetylation positively affects the latter binding to the ARE in a promoter-specific manner in mammary epithelial cells exposed to arsenite (9). In contrast, NRF2 deacetylation has been shown to decrease the levels of nuclear NRF2 and induction of its target gene expression (14). Although we found increased levels of NRF2 accumulation in sirtinol-treated cells under the basal condition, our coimmunoprecipitation assays revealed no striking difference in the levels of acetylated NRF2 between the vehicle- and sirtinoltreated cells under basal or chronic hyperoxic conditions (Figure 6). NRF2 acetylation is detectable in cells exposed to acute $(6 \mathrm{~h})$ hyperoxia, but these levels are returned to nearly basal levels after 12 hours of hyperoxia, and they are minimally detectable in cells exposed to chronic hyperoxia. Because acetylation is a dynamic and reversible process, it is possible that NRF2 is acetylated transiently or that only a few NRF2 molecules are modified in chronic hyperoxia, which were undetectable in our experimental conditions. In addition, it is unclear why acetylated NRF2 levels are low in sirtinol-treated cells exposed to acute hyperoxia; this warrants further investigation.

Our results show that sirtinol treatment increases the stability of NRF2 and NRF2-dependent gene expression under the basal condition. These results are in line with those of previous studies showing augmented and reduced levels of NRF2-dependent AOE expression in cells treated with SIRT1 inhibitors (e.g., EX-527 and nicotinamide) and a SIRT1 activator (e.g., resveratrol), respectively (14).

However, unlike sirtinol, siRNA-mediated SIRT1 depletion did not affect both NRF2 protein stability and NRF2-dependent AOE expression under the basal condition (Figure 3). However, SIRT1-depletion reduced cell death and NRF2-dependent AOE expression in chronic hyperoxia in a manner similar to that of sirtinol. Previous studies have shown that acetylation does not affect the stability of the NRF2 protein (9). Thus, it is conceivable that sirtinol directly or indirectly modulates/disrupts KEAP1-NRF2 interactions and thereby increases NRF2's stability and its target gene expression. Otherwise, it is possible that SIRT2 is able to compensate for SIRT1 in suppressing NRF2 activation under the basal condition.

It is noteworthy that, despite elevated levels of nuclear NRF2, not all NRF2 target gene expression was up-regulated by either inhibition or depletion of SIRT1. This result suggests that SIRT1 selectively regulates NRF2-dependent AOE expression. Previous studies have detected acetylated NRF2 on only a subset of promoters of NRF2 target genes, and the authors have proposed that acetylation of NRF2 may be required for fine-tuning and/or augmenting DNA binding and promoter transactivation, rather than being a switch-on or switch-off mechanism (9). Indeed, our results showed decreased levels of 
NRF2-dependent AOE expression-but not a complete lack-in both SIRT1-inhibited and -depleted cells exposed to hyperoxia (Figures 1 and 3). Previous studies have shown that reduced levels of SIRT1 in the lungs of mice exposed to cigarette smoke are accompanied by acetylation and degradation of the FOXO3

transcription factor and airspace enlargement (28). In contrast, we found an increased expression of SIRT1 in the lungs of mice exposed to chronic hyperoxia, and sirtinol attenuated hyperoxia-induced lung alveolar permeability and toxicity in vivo (Figure 7 ), suggesting that SIRT1 promotes hyperoxia-induced lung epithelial cell injury and death; thus, it appears that SIRT1 is distinctly induced in lung cell types by prooxidant insults and exerts different functions in a contextual manner.

\section{Conclusions}

In summary, our studies reveal SIRT1 to be a critical positive regulator of lung epithelial cell death, and we have shown that it mediates this regulation by altering the balance between proand anti-apoptotic proteins and not by attenuating NRF2-dependent AOE transcription in chronic hyperoxia. We propose that either depletion of SIRT1 or dampening of its activity may be useful in mitigating cell death in chronic hyperoxia.

Author disclosures are available with the text of this article at www.atsjournals.org.

\section{References}

1. Rubenfeld GD, Caldwell E, Peabody E, Weaver J, Martin DP, Neff M, Stern EJ, Hudson LD. Incidence and outcomes of acute lung injury. $N$ Engl $J$ Med 2005;353:1685-1693.

2. Cho HY, Reddy SP, Yamamoto M, Kleeberger SR. The transcription factor NRF2 protects against pulmonary fibrosis. FASEB $J$ 2004;18: 1258-1260.

3. Cho HY, Jedlicka AE, Reddy SPM, Kensler TW, Yamamoto M, Zhang LY, Kleeberger SR. Role of NRF2 in protection against hyperoxic lung injury in mice. Am J Respir Cell Mol Biol 2002;26:175-182.

4. Reddy NM, Kleeberger SR, Kensler TW, Yamamoto M, Hassoun PM, Reddy SP. Disruption of Nrf2 impairs the resolution of hyperoxia-induced acute lung injury and inflammation in mice. J Immunol 2009;182:7264-7271.

5. Cho HY, Reddy SP, Kleeberger SR. Nrf2 defends the lung from oxidative stress. Antioxid Redox Signal 2006;8:76-87.

6. Reddy NM, Potteti HR, Mariani TJ, Biswal S, Reddy SP. Conditional deletion of Nrf2 in airway epithelium exacerbates acute lung injury and impairs the resolution of inflammation. Am J Respir Cell Mol Biol 2011;45:1161-1168.

7. Taguchi K, Motohashi H, Yamamoto M. Molecular mechanisms of the Keap1-Nrf2 pathway in stress response and cancer evolution. Genes Cells 2011;16:123-140.

8. Salazar M, Rojo Al, Velasco D, de Sagarra RM, Cuadrado A. Glycogen synthase kinase-3beta inhibits the xenobiotic and antioxidant cell response by direct phosphorylation and nuclear exclusion of the transcription factor Nrf2. J Biol Chem 2006;281:14841-14851.

9. Sun Z, Chin YE, Zhang DD. Acetylation of Nrf2 by p300/CBP augments promoter-specific DNA binding of Nrf2 during the antioxidant response. Mol Cell Biol 2009;29:2658-2672.

10. Houtkooper RH, Pirinen E, Auwerx J. Sirtuins as regulators of metabolism and healthspan. Nat Rev Mol Cell Biol 2012;13:225-238.

11. Sebastián C, Satterstrom FK, Haigis MC, Mostoslavsky R. From sirtuin biology to human diseases: an update. J Biol Chem 2012;287: 42444-42452.

12. McBurney MW, Clark-Knowles KV, Caron AZ, Gray DA. Sirt1 is a highly networked protein that mediates the adaptation to chronic physiological stress. Genes Cancer 2013;4:125-134.

13. Mercado N, Thimmulappa R, Thomas CM, Fenwick PS, Chana KK, Donnelly LE, Biswal S, Ito K, Barnes PJ. Decreased histone deacetylase 2 impairs Nrf2 activation by oxidative stress. Biochem Biophys Res Commun 2011;406:292-298.

14. Kawai Y, Garduño L, Theodore M, Yang J, Arinze IJ. Acetylationdeacetylation of the transcription factor Nrf2 (nuclear factor erythroid 2-related factor 2) regulates its transcriptional activity and nucleocytoplasmic localization. J Biol Chem 2011;286:7629-7640.

15. Piao CQ, Liu L, Zhao YL, Balajee AS, Suzuki M, Hei TK. Immortalization of human small airway epithelial cells by ectopic expression of telomerase. Carcinogenesis 2005;26:725-731.

16. Moehlenkamp JD, Johnson JA. Activation of antioxidant/electrophileresponsive elements in IMR-32 human neuroblastoma cells. Arch Biochem Biophys 1999;363:98-106.
17. Potteti HR, Reddy NM, Hei TK, Kalvakolanu DV, Reddy SP. The nrf2 activation and antioxidative response are not impaired overall during hyperoxia-induced lung epithelial cell death. Oxid Med Cell Longev 2013;2013:798401.

18. Mantell LL, Lee PJ. Signal transduction pathways in hyperoxiainduced lung cell death. Mol Genet Metab 2000;71:359-370.

19. Buccellato LJ, Tso M, Akinci OI, Chandel NS, Budinger GR. Reactive oxygen species are required for hyperoxia-induced Bax activation and cell death in alveolar epithelial cells. J Biol Chem 2004;279:6753-6760.

20. llizarov AM, Koo HC, Kazzaz JA, Mantell LL, Li Y, Bhapat R, Pollack S, Horowitz S, Davis JM. Overexpression of manganese superoxide dismutase protects lung epithelial cells against oxidant injury. $A m \mathrm{~J}$ Respir Cell Mol Biol 2001;24:436-441.

21. Wispé JR, Warner BB, Clark JC, Dey CR, Neuman J, Glasser SW, Crapo JD, Chang LY, Whitsett JA. Human Mn-superoxide dismutase in pulmonary epithelial cells of transgenic mice confers protection from oxygen injury. J Biol Chem 1992;267: 23937-23941.

22. Folz RJ, Abushamaa AM, Suliman HB. Extracellular superoxide dismutase in the airways of transgenic mice reduces inflammation and attenuates lung toxicity following hyperoxia. J Clin Invest 1999; 103:1055-1066.

23. Koo HC, Davis JM, Li Y, Hatzis D, Opsimos H, Pollack S, Strayer MS, Ballard PL, Kazzaz JA. Effects of transgene expression of superoxide dismutase and glutathione peroxidase on pulmonary epithelial cell growth in hyperoxia. Am J Physiol Lung Cell Mol Physiol 2005;288:L718-L726.

24. Budinger GR, Tso M, McClintock DS, Dean DA, Sznajder JI, Chandel NS. Hyperoxia-induced apoptosis does not require mitochondrial reactive oxygen species and is regulated by $\mathrm{Bcl}-2$ proteins. J Biol Chem 2002;277:15654-15660.

25. Li Y, Xu W, McBurney MW, Longo VD. SirT1 inhibition reduces IGF-I/IRS-2/Ras/ERK1/2 signaling and protects neurons. Cell Metab 2008;8:38-48.

26. Chen S, Xiao X, Feng X, Li W, Zhou N, Zheng L, Sun Y, Zhang Z, Zhu W. Resveratrol induces Sirt1-dependent apoptosis in 3T3-L1 preadipocytes by activating AMPK and suppressing AKT activity and survivin expression. J Nutr Biochem 2012;23: 1100-1112.

27. Rajamohan SB, Pillai VB, Gupta M, Sundaresan NR, Birukov KG, Samant S, Hottiger MO, Gupta MP. SIRT1 promotes cell survival under stress by deacetylation-dependent deactivation of poly (ADP-ribose) polymerase 1. Mol Cell Biol 2009;29:4116-4129.

28. Yao H, Chung S, Hwang JW, Rajendrasozhan S, Sundar IK, Dean DA, McBurney MW, Guarente L, Gu W, Rönty M, et al. SIRT1 protects against emphysema via $\mathrm{FOXO}-$ mediated reduction of premature senescence in mice. J Clin Invest 2012;122:2032-2045.

29. Tanno M, Kuno A, Yano T, Miura T, Hisahara S, Ishikawa S, Shimamoto K, Horio Y. Induction of manganese superoxide dismutase by nuclear translocation and activation of SIRT1 promotes cell survival in chronic heart failure. J Biol Chem 2010; 285:8375-8382. 